\title{
Speckle observations of double and multiple stars at Pic du Midi: Measurements during 1995 and 1997 and new orbits ${ }^{\star, \star \star, \star \star \star}$
}

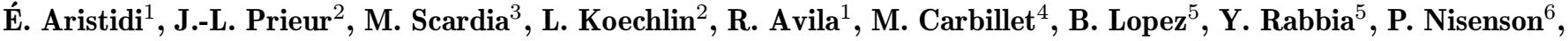 \\ and D. Gezari ${ }^{6}$ \\ 1 UMR 6525 Astrophysique, Université de Nice Sophia, Antipolis, Centre National de la Recherche Scientifique, Parc Valrose, \\ 06108 Nice Cedex 2, France \\ 2 Observatoire Midi Pyrénées, 14 Av. Edouard Belin, 31400 Toulouse, France \\ 3 Osservatorio Astronomico di Brera, Via E. Bianchi 46, 22055 Merate, Italy \\ 4 Osservatorio Astrofisico di Arcetri, Largo Enrico Fermi 5, 50125 Firenze, Italy \\ 5 UMR 6528, Département Fresnel de l'Observatoire de la Côte d'Azur, BP. 4229, 06304 Nice Cedex 4, France \\ ${ }^{6}$ Center for Astrophysics, 60 Garden Street, Cambridge MA 02138, U.S.A.
}

Received May 27; accepted September 4, 1998

\begin{abstract}
We present speckle observations of 48 double and multiple stars observed with the 2-meter "Télescope Bernard Lyot" (TBL) in December 1995, January 1997 and June 1997. Angular separations, absolute position angles and relative photometry result from these observations. New orbital elements have been recalculated for 8 double stars.
\end{abstract}

Key words: techniques: interferometric — binaries: close

\section{Introduction}

This paper presents the results of double and multiple star speckle observations performed with the $2 \mathrm{~m}$ Télescope Bernard Lyot of the Pic du Midi observatory (France). Instrumentation and data analysis were partly described in a previous paper (Aristidi et al. 1997b). During the run of June 1997, we tested a real time system described in Sect. 2. Five stars could also be observed using the PAPA photon-counting detector (Papaliolios et al. 1982, 1985).

Double stars measured here are either long-period binaries for which the last observation is old, or stars previously observed by us and for which the companion's posi-

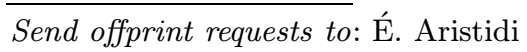

* Based on observations made with $2 \mathrm{~m}$ Télescope Bernard Lyot, Pic du Midi, France.

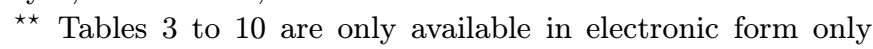
via the CDS at ftp to cdsarc.u-strasbg.fr (130.79.128.5) or via http://cdsweb.u-strasbg.fr/Abstract.html

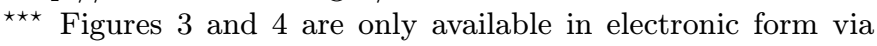
http://edpsciences.com tion was measured far from the predicted value (Aristidi et al. 1997b). Some stars were also selected because their orbit parameters are very uncertain. Two of these double stars were recently discovered as double: MOAI 1 (SAO 12917) observed in December 1995 (Carbillet et al. 1996, 1998b), and $\nu$ Cyg discovered by Hipparcos (The Hipparcos Catalogue, 1997). Multiple stars were selected from the Multiple Star Catalogue (Tokovinin 1997). All of them present a lack of measurement: unknown orbits, unknown spectral classes of the components, companion suspected from other observational techniques (spectroscopy). These stars were selected together with A.A. Tokovinin.

\section{Observations}

Data were recorded during three observing runs in December 1995, January 1997 and June 1997 at the TBL. We had quite poor weather conditions with very short coherence times of a few milliseconds only and a FWHM seeing often larger than 2 arcsec. A total of 33 double stars and 15 multiple stars was observed; details are given in Table 1. The focal instrumentation is the speckle camera of the Aperture Synthesis group of Observatoire MidiPyrénées (OMP). For the major part of the nights it was coupled with the ICCD detector of Nice University. This instrumentation is described in Aristidi et al. (1997b) and Prieur et al. (1998). During the runs in January 1995 and January 1997, the images were recorded on video tape for further processing. In June they were also sent to a PC hosting a Matrox Genesis digitizer board equipped with a digital signal processor (C80) which enables near realtime processing. As an example the power spectrum for 
Table 1. Table of measurements. $\lambda$ denotes the wavelength and $\Delta \lambda$ the bandwidth. The column labelled Mult. gives the number of stars in the system (multiplicity). The column labelled Obj. give the name of the measured components of the multiple star. Predicted values of $\rho$ and $\theta$ are computed from the latest available orbits. For orbits prior to 1982 (followed by a $\star$ ), orbital elements were found in the catalogue of Worley \& Heintz, 1983. For $\epsilon$ Hya the angular separation ABxC was computed from $\mathrm{AB}, \mathrm{AC}$ and $\Delta m_{A B}$ as the distance between $\mathrm{C}$ and the photocenter of $\mathrm{AB}$. The columns labelled $\Delta \rho$ and $\Delta \theta$ give the residuals in $\rho$ and $\theta$

\begin{tabular}{|c|c|c|c|c|c|c|c|c|c|c|c|c|c|c|c|c|c|c|c|c|c|}
\hline है & & & & & 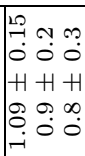 & & & 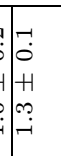 & 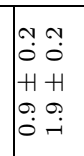 & & & \begin{tabular}{c}
\multirow{2}{*}{} \\
0 \\
+1 \\
0 \\
0 \\
-1
\end{tabular} & & & & & $\begin{array}{l}-1 \\
0 \\
H \\
0 \\
-1\end{array}$ & & & & \\
\hline & & $\sigma_{2}$ & & $\mid \stackrel{-1}{\infty}$ & $\stackrel{\infty}{\rightarrow-}$ & 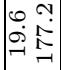 & 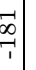 & $\mid$ & $\mid \begin{array}{ll}0 \\
\dot{i} & 0 \\
i & 2\end{array}$ & & {$\left[\begin{array}{ll}\infty & 0 \\
\infty & 0 \\
1 & 0\end{array}\right.$} & & 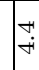 & & نُ & $\begin{array}{l}\infty \\
i \infty \\
\dot{2}\end{array}$ & $\tilde{p}_{i}$ & & $\stackrel{-}{-}$ & & $\begin{array}{l}20 \\
9 \\
9\end{array}$ \\
\hline & & $\begin{array}{ll}2 & \infty \\
\infty & \infty \\
- & \infty \\
-\end{array}$ & & în & $\begin{array}{l}\stackrel{+}{0} \\
\stackrel{\vec{N}}{N} \\
\end{array}$ & 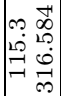 & $\begin{array}{c}\infty \\
\infty \\
\infty \\
\infty \\
\sim\end{array}$ & & 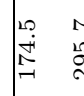 & 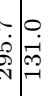 & 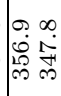 & & تِ & & 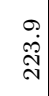 & ?ִ & 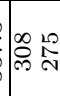 & & $\begin{array}{l}0 \\
\infty \\
\tilde{N} \\
\tilde{N}\end{array}$ & & ثُ \\
\hline & 8 & 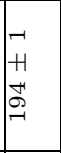 & 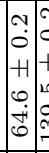 & 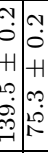 & $\begin{array}{l}\text { Na } \\
0 \\
+ \\
0 \\
0 \\
\stackrel{N}{N} \\
\stackrel{N}{*}\end{array}$ & 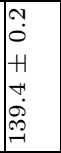 & 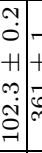 & 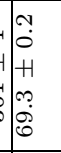 & 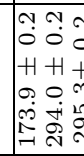 & 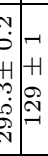 & $\begin{array}{l}\tilde{N} \\
0 \\
H \\
+ \\
-\dot{\infty} \\
\infty \\
\infty \\
\infty\end{array}$ & 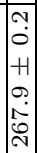 & 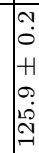 & 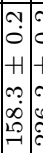 & 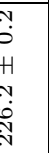 & 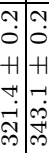 & $\begin{array}{l}-1 \\
+H \\
C \\
N\end{array}$ & $\begin{array}{l}\mathcal{N} \\
\tilde{O} \\
H \\
H \\
\tilde{N} \\
\stackrel{N}{N} \\
\tilde{N}\end{array}$ & 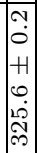 & & $\begin{array}{l}-1 \\
+ \\
\sim \\
-1 \\
-1 \\
-1\end{array}$ \\
\hline & & $\begin{array}{lll} & 0 \\
0 & 0 \\
0 & 0 & 0\end{array}$ & & : & $\begin{array}{l}\qquad 0 \\
0 \\
0 \\
1 \\
1\end{array}$ & $\begin{array}{l}0 \\
-1 \\
-1 \\
0\end{array}$ & $\begin{array}{l}0 \\
0 \\
0 \\
1 \\
1\end{array}$ & 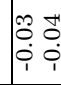 & 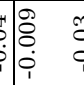 & 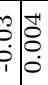 & $\begin{array}{ll}1 & 0 \\
10 & 8 \\
0 & 0 \\
0 & 0\end{array}$ & & & & $\begin{array}{lll}8 & 71 \\
0 & 0 & 0 \\
0 & 0 & 0 \\
0 & 0\end{array}$ & 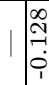 & $\mid \begin{array}{ll}0 & 0 \\
0 & 0 \\
0 & 0 \\
0 & 0\end{array}$ & & $\begin{array}{l} \\
0 \\
0 \\
0\end{array}$ & & 尊 \\
\hline $\begin{array}{l}\vec{\Xi} \Xi \\
0 \\
0\end{array}$ & & 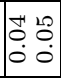 & 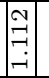 & : & 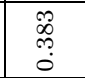 & 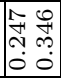 & 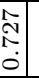 & & $\begin{array}{ll}\infty \\
\\
N & 1 \\
0 & 0 \\
0 & 0\end{array}$ & 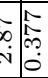 & {$\left[\begin{array}{l}0 \\
0 \\
0 \\
0 \\
0 \\
0 \\
0 \\
0\end{array}\right.$} & & & $\mid$\begin{tabular}{|c|}
$-\infty$ \\
0 \\
20 \\
0 \\
0
\end{tabular} & \begin{tabular}{l}
0 \\
\multirow{7}{*}{} \\
0
\end{tabular} & 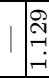 & $\mid \begin{array}{ll}2 & = \\
0 & 7 \\
0 & -7\end{array}$ & & 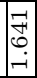 & & $\begin{array}{l}\text { os } \\
\text { N } \\
0 \\
0\end{array}$ \\
\hline & $\begin{array}{l}H \\
+ \\
\infty \\
0 \\
0\end{array}$ & $\begin{array}{l}1 \\
0 \\
0 \\
H \\
H \\
\infty \\
0 \\
0 \\
0\end{array}$ & 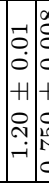 & 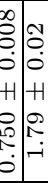 & 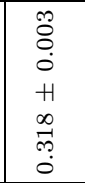 & 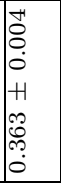 & 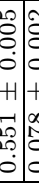 & 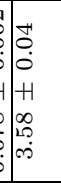 & 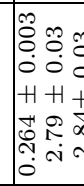 & 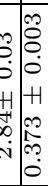 & \begin{tabular}{l}
$\vec{D}$ \\
0 \\
0 \\
0 \\
$H$ \\
$H$ \\
$\qquad$ \\
0 \\
\hdashline \\
0 \\
0
\end{tabular} & $\begin{array}{l} \\
0 \\
\dot{0} \\
+1 \\
0 \\
0 \\
0 \\
0\end{array}$ & $\begin{array}{l}0 \\
0 \\
H \\
+1 \\
\ddot{0} \\
\dot{\theta} \\
-1\end{array}$ & 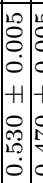 & 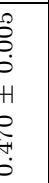 & 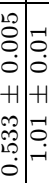 & 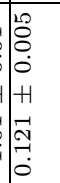 & 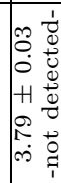 & $\begin{array}{l}- \\
0 \\
0 \\
+1 \\
+1 \\
0 \\
0 \\
-1\end{array}$ & & 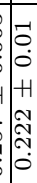 \\
\hline 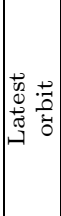 & & 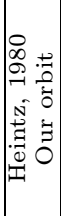 & 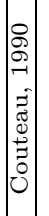 & 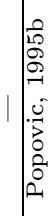 & 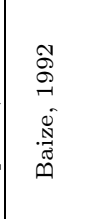 & 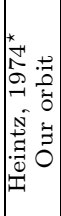 & 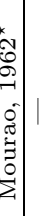 & 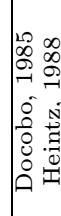 & 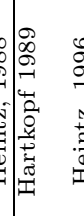 & ل & 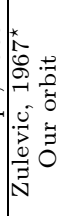 & & 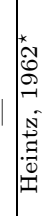 & & 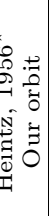 & : & 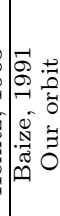 & & 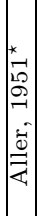 & & 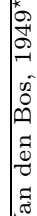 \\
\hline 20 & & $\begin{array}{lll}\frac{m}{4} & 0 \\
\end{array}$ & & $\frac{9}{4}$ & & & $\oplus$ & $\frac{n}{2}$ & 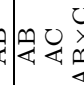 & & & 这 & & & $\frac{9}{4}$ & & $\frac{m}{4} \frac{0}{4}$ & O & & & \\
\hline 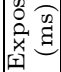 & $\infty$ & 0 & & 0 & 웅 & $\infty$ & $\infty$ & $\mid \neg$ & סL 10 & $\underline{0}$ & N & & $\stackrel{0}{-1}$ & & - & & A & $\stackrel{\leftrightarrow}{\sim} N$ & $\infty$ & & 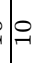 \\
\hline 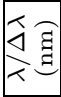 & $\begin{array}{l}1 \\
10 \\
0 \\
0 \\
0 \\
0\end{array}$ & 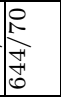 & 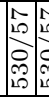 & 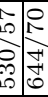 & 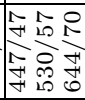 & & & $\hat{n}$ & 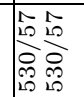 & $\begin{array}{l}R \\
\text { R } \\
\text { fit } \\
0\end{array}$ & 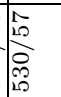 & & 6 & & & 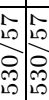 & $\left\{\begin{array}{l}1 \\
10 \\
2 \\
0 \\
0 \\
0 \\
0\end{array}\right.$ & 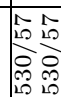 & & & $\begin{array}{l}R \\
F \\
0\end{array}$ \\
\hline 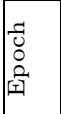 & 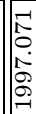 & 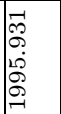 & 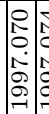 & 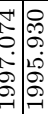 & 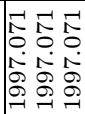 & 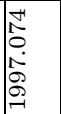 & 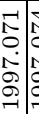 & 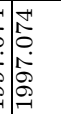 & 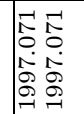 & 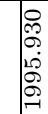 & 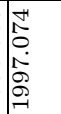 & $\begin{array}{l}\text { ㅌ. } \\
0 \\
0 \\
0 \\
0\end{array}$ & 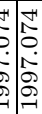 & 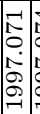 & & 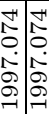 & 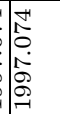 & 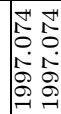 & 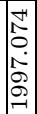 & & 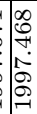 \\
\hline 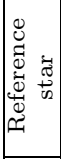 & 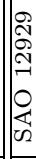 & ‡ & 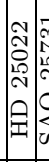 & 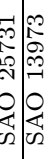 & $\mid \begin{array}{l}8 \\
8 \\
8 \\
18 \\
0 \\
0 \\
0 \\
4 \\
0\end{array}$ & 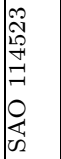 & \begin{tabular}{c|c}
0 \\
0 \\
0 \\
0 \\
2 \\
0 \\
0 \\
0
\end{tabular} & $\begin{array}{l}a \\
\tilde{J} \\
0 \\
0 \\
0\end{array}$ & 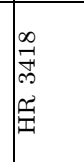 & & 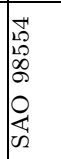 & & $\infty$ & & & 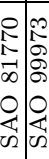 & 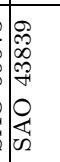 & & & 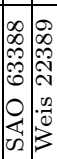 & $\begin{array}{l}R \\
0 \\
0 \\
0 \\
0 \\
0 \\
\theta \\
\theta\end{array}$ \\
\hline$\stackrel{ \pm}{\exists}$ & N & & ค & $m$ & N & A & & 40 & 20 & N & N & & N & & & $\curvearrowright$ N & 10 & & N & & N \\
\hline 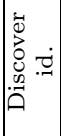 & 茟 & 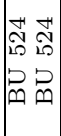 & 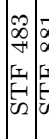 & 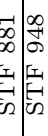 & 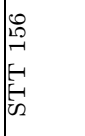 & 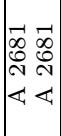 & & 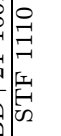 & 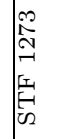 & $\begin{array}{l}\hat{N} \\
\infty \\
-1\end{array}$ & 孞点 & & 恿 & & 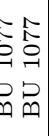 & 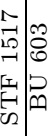 & 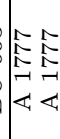 & & & 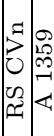 & $\left\{\begin{array}{l}2 \\
20 \\
10\end{array}\right.$ \\
\hline 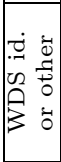 & 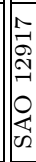 & 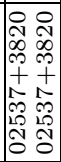 & 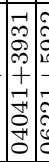 & 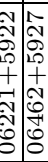 & & 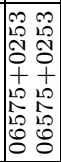 & & 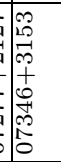 & 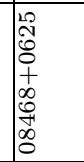 & $\begin{array}{l}\vec{F} \\
\forall \\
+ \\
\infty \\
8 \\
8\end{array}$ & 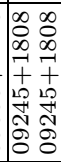 & & & & 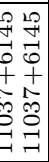 & 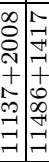 & 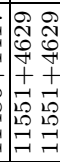 & 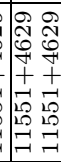 & 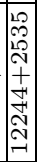 & 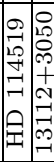 & 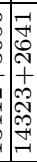 \\
\hline
\end{tabular}


Table 1. Table of measurements (continued). For observations made with the PAPA detector, a $\dagger$ has been added in superscript to the exposure time (usually $2 \mathrm{~ms}$ ). $\left(^{*}\right) \nu$ Cyg was in fact discovered as double and then not used as reference star in the processing of BU 151

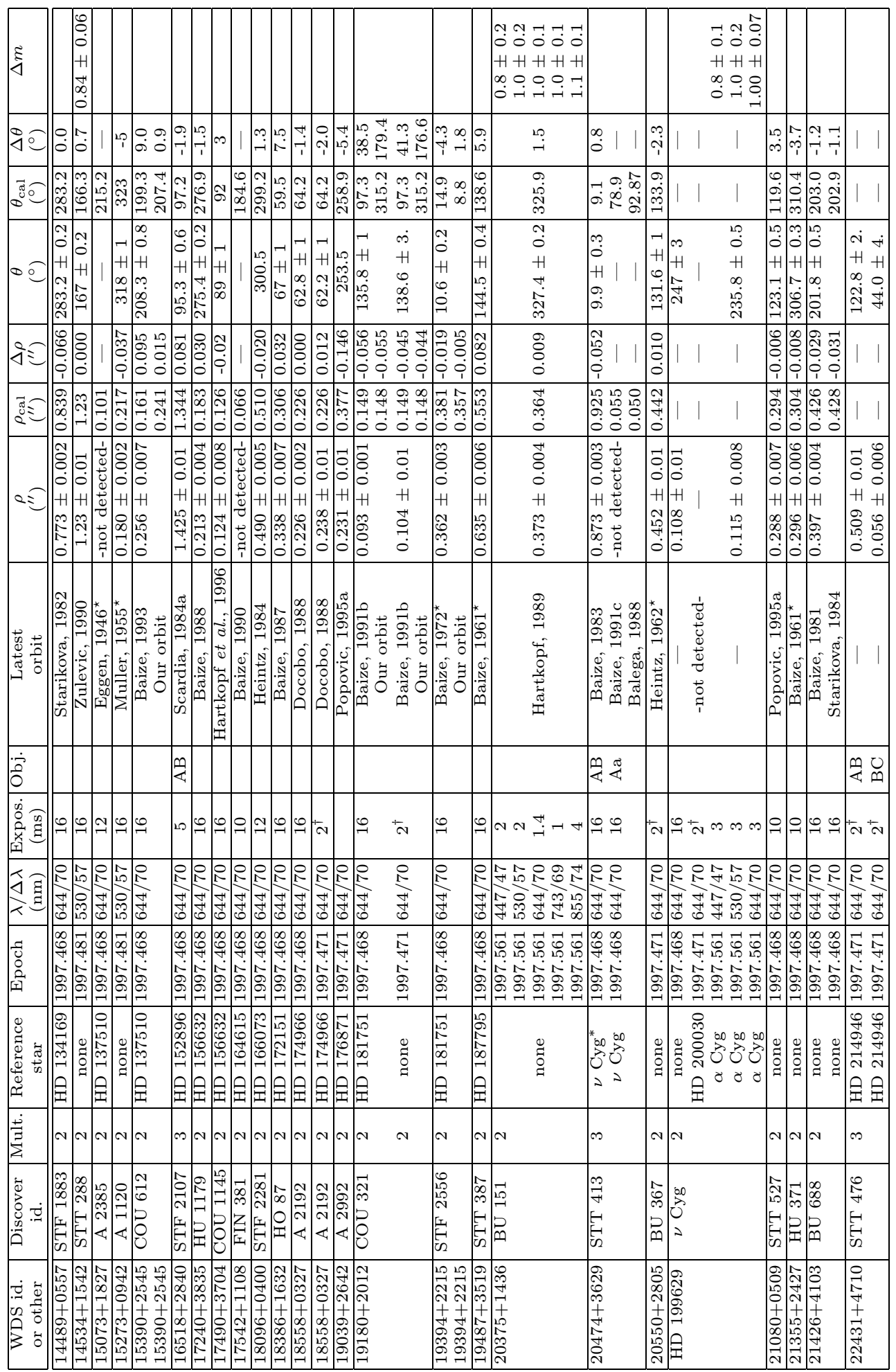


a $128 \times 128$ image size is computed at a rate of about 20 frames per second. The use of a reference star for usual calibration of the telescope+atmosphere transfer function was sometimes avoided by computing the cross-correlation between time-separated images and subtracting it from the autocorrelation (Worden et al. 1977). Though at a lower speed (9 frames/s) the system is also programmed to compute the cross correlation between the images and their square in order to find the absolute position angles of binaries (Aristidi et al. 1997a). This system does not include yet the classification of images according to the seeing that is used in the data processing as described in Aristidi et al. (1997b). Bright stars $\left(m_{V} \lesssim 6\right)$ were re-processed from the video tape for magnitude difference determination using probability imaging (Carbillet et al. 1998a). This provides also the absolute position angle (PA) and is useful to confirm the PA computed by the cross-correlation technique (Aristidi et al. 1997a).

Problem of saturation is took into account the following way: before recording frames, the video digitizer displays saturated pixels, allowing the observer to adjust the gain and the offset of the detector to reduce the saturation. Moreover, at processing time (probability imaging), pixels at the maximum intensity level are rejected from the calculation of the magnitude difference.

The PAPA camera was used during part of these observations but because of technical testing and bad weather conditions it could only lead to measurements on the night of $23 / 06 / 97$. It is actually a new version of the original camera described in Papaliolios et al. (1982, 1985). Modifications have been jointly implemented by P. Nisenson (Harvard Center for Astrophysics), D. Gezari (NASA) and L. Koechlin (OMP) in the last five years. The current version has a new binary mask setup and a refurbished image intensifier. A problem in the regulation of the high voltage power supply caused a strong geometric distorsion and a variation of the overall scale which imposed quasi permanent scale calibrations during the night. A small "hole" at the center of the autocorrelation function was also noticed, due to a lack of detectivity of the electronics after each photo-event detection. A whole reduction procedure had to be set up in order to correct for these defects:

- Each star image was corrected with the distortion and scaling coefficients interpolated from the two images of the calibrating grid which were taken immediately before and after the star observation.

- To remove the hole at the center of the autocorrelation, each photo-event was correlated with the photo-events of a "gliding" time window located at $1 \mathrm{msec}$ after the arrival time of the photo-event. The very poor atmospheric conditions did not allow us to use a time window larger than $2 \mathrm{msec}$ (as the typical coherence time was less than $3 \mathrm{msec}$ ).

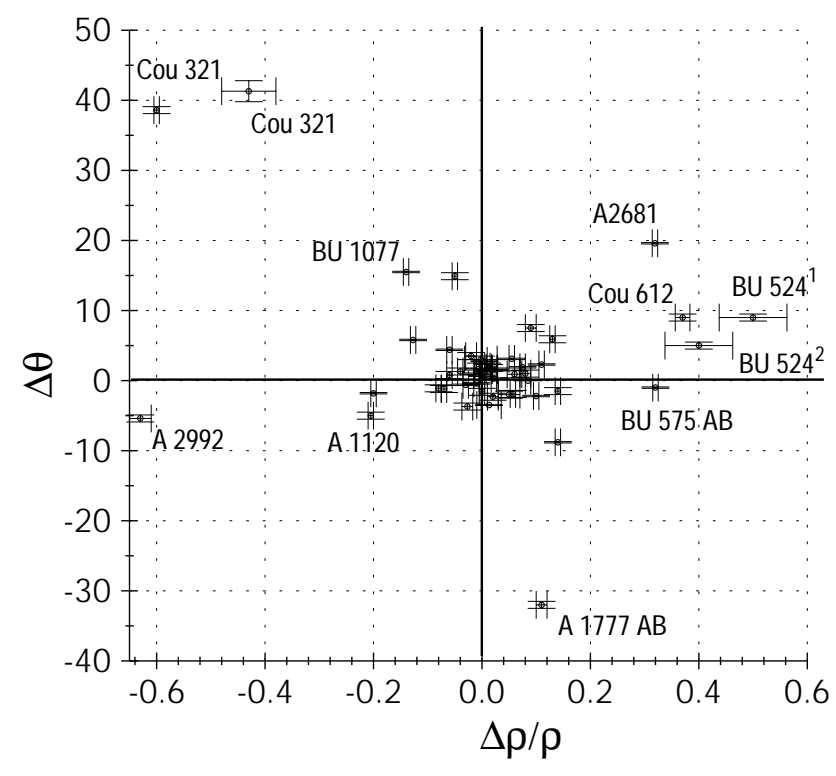

Fig. 1. Two dimensionnal plot of the residuals in position angle $(\Delta \theta)$ and os the relative residuals in separation $(\Delta \rho / \rho)$. For couples with known orbit, each measurement corresponds to a point in the graph. Error bars in $\rho$ and $\theta$ have been drawn to scale. For points with large residuals, corresponding star names are given (in the case of BU 524, ${ }^{1}$ refers to the orbit of 1980 while ${ }^{2}$ refers to our orbit)

\section{Results and discussion}

The measurements are presented in Table 1. For multiple systems with at least 3 components, the name of each measured couple is given. Ephemeris are computed for double and/or multiple stars with known orbits. Figure 1 displays the residuals in $\rho$ and $\theta$ as a cloud of points with error bars in the $(\Delta \rho / \rho, \Delta \theta)$ plane. This cloud of points centered on $(0,0)$ validates the data reduction and calibration procedure. We also notice a few cases of abnormally large differences between our measurements and the expected values from the previously determined orbits. This is the case for example of Cou $321\left(\Delta \theta \simeq 40^{\circ}\right)$ or A $2992\left(\frac{\Delta \rho}{\rho} \simeq 60 \%\right)$. Corresponding points are labelled with star names in Fig. 1. In order to check whether the problem comes from a poor observation or a bad orbit, we looked for other published data (Hartkopf 1998) and plotted residuals as a function of time. This was done for nine stars (BU 524, A 2681, BU 575, A 2477, BU 1077, A 1777, Cou 612, Cou 321, and STF 2556). Examination of these curves led us to recalculate new orbital elements for all these stars but BU 575 .

The following comments can be made for some individual stars:

- Moaï 1 was discovered as double in December 1995 (Carbillet et al. 1996). An error occurred with the published values of the position angle, the correct values being: date $=1995.947, \rho=0$ ". $110 \pm 0$ ! $003, \theta=167 \pm 1^{\circ}$ (Carbillet et al. 1998b). 
- BU 524 was observed near periastron, close to the limiting resolution of the telescope $(0$ '. 066 mas at $\lambda=$ $644 \mathrm{~nm})$.

- A 1777 is a quintuple star. AB, AC and $\mathrm{AD}$ are expected to be resolved by a $2 \mathrm{~m}$ telescope. The couple $\mathrm{AC}$ did not fit in our field of view (separation $\simeq 600^{\prime \prime}$ ), so we did not measure it. We searched for companions close to star $\mathrm{D}$ but nothing was detected (for a separation $\geq 0$.'05 and a magnitude difference $<5)$.

- STF 1517 is a double star with a very uncertain orbit (Hopmann 1970), computed period is about $4050 \mathrm{yr}$. This orbit cannot be used for ephemeris computation.

$-\nu$ Cyg was initially observed as a reference star and discovered double. The first observation (1997.468) being very poor, it has been reobserved in 1997.471 for confirmation and in 1997.561 for three colors relative photometry. Its duplicity was discovered by Hipparcos (The Hipparcos Catalogue, 1997).

- BU 575 has a very uncertain orbit. A possible confusion between stars A and B (small magnitude difference) causes an uncertainty of $180^{\circ}$.

- XY Leo is mentioned as a quadruple star in Tokovinin (1997). Spectroscopic observations have revealed that this system is composed of two close pairs (denoted as Aab and Bab), one being a contact binary. The expected separation between the pairs deduced from spectrophotometric measurements is $A B \simeq 0$ !' 15 (Barden 1987). To our knowledge, it has never been resolved by visible speckle interferometry. We also did not detect this companion.

\section{Orbit calculation}

The orbits were computed using the method of Kowalsky (1873) and Hellerich (1925). The error bars calculation is described in Scardia (1983) for errors on the orbital elements, and in Scardia (1984b) for errors on the sum of masses of the stars.

The orbital elements are presented below for each couple. WDS identification (equinox 2000) (Mason 1998) is given for each star together with discover name and ADS catalog number. Orbits are plotted in Fig. 3 and Fig. 4. History of all measurements for each star are presented at the end of the paper. We computed eight orbits. Two of them may be considered as reliable (BU 524 and A 1777); a mass estimation and detailed dicussion is given below. Th six others are premature and the orbital elements are summarised in Table 3.

- WDS 02537+3820 - BU 524 - ADS 2200 AB: This close double star (separation $\simeq 0^{\prime \prime} .1$ ) with $\Delta m \simeq 1$ is a difficult object for visual observations. This explains the large residuals on the orbit plot. 160 measurements have been published since 1878, 129 have been retained for the orbit computation. On the plot of the new orbit one can see the lack of data near the periastron where the predicted separation is under $0{ }^{\prime \prime} 1$. In such a case this star is a good target dedicated for speckle interferometry techniques as demonstrated by the three points near periastron (two values comes from the CHARA, the third is ours). According to our orbit, the next periastron will occur in October 2027.

\begin{tabular}{lccc}
\hline Orbital elements & & & \\
\hline \hline$\Omega\left(^{\circ}\right)(2000.0)$ & 28.87 & \pm & 1.4 \\
$\omega\left(^{\circ}\right)$ & 266.78 & \pm & 0.6 \\
$i\left(^{\circ}\right)$ & 115.8 & \pm & 1.9 \\
$P(\mathrm{yr})$ & 31.572 & \pm & 0.50 \\
$T(\mathrm{yr})$ & 1964.651 & \pm & 0.055 \\
$e$ & 0.8067 & \pm & 0.017 \\
$a$ & $00^{\prime \prime} 253$ & \pm & 0.022 \\
\hline Astrophysical Information & & \\
\hline \hline mag & $5.34-6.35 \mathrm{Sp}$ & $\mathrm{F} 4 \mathrm{IV}$ \\
Hipparcos parallax & $0^{\prime \prime} 01387$ & \pm & $0^{\prime \prime} 00086$ \\
Semi-major axis $(\mathrm{AU})$ & 18.2 & \pm & 2.7 \\
Sum of masses $\left(\mathcal{M}_{\odot}\right)$ & 6.09 & \pm & 2.75 \\
\hline \multicolumn{4}{c}{}
\end{tabular}

Dynamic parallax (Couteau 1978) could not be computed since star A is not a main-sequence star (spectral class F4IV). This does not agree with published masses of stars A and B estimated from their spectral classes (Tokovinin 1997): $\mathcal{M}_{A}=1.38 \mathcal{M}_{\odot}$ and $\mathcal{M}_{B}=1.15 \mathcal{M}_{\odot}$. If we suppose that the spectral class F4IV for the primary is well estimated, it might be possible that this system contains other stars to be discovered.

Van den Bos (1938) calculated two sets of orbital elements, a short period one $(P=31.6 \mathrm{yr})$ and a long period one $(P=63.1 \mathrm{yr})$, the second being justified by quadrant uncertainties and the fact that the observed separation was always greater than 0.1 , whereas the short-period orbit predicted a very close periastron of about 0. . 002 . We then computed a long period orbit with the following orbital elements: $T=1988.066$, $P=62.851 \mathrm{yr}, e=0.0286, a=0^{\prime \prime} .211, \Omega=129.79^{\circ}$, $\omega=51.47^{\circ}, i=133.73^{\circ}$. Residuals in separation and in position angle are plotted in Fig. 2 as a function of time. As can be shown on this figure and on the orbit plot, this long period orbit does not fit the observations close to the periastron when the star velocities are high (especially the last speckle measurements). From an astrophysical point of view, the total mass of the system given by this orbit is too small $\left(0.89 \mathcal{M}_{\odot}\right)$. This long period orbit is then to be rejected.

\begin{tabular}{|ccc|ccc|}
\hline \multicolumn{6}{|c|}{ Ephemeris } \\
\hline \hline Date & $\rho\left(^{\prime \prime}\right)$ & $\theta\left(^{\circ}\right)$ & Date & $\rho\left(^{\prime \prime}\right)$ & $\theta\left(^{\circ}\right)$ \\
\hline 1999.000 & .165 & 7.6 & 2004.000 & .206 & 341.9 \\
2000.000 & .181 & 1.3 & 2005.000 & .207 & 337.5 \\
2001.000 & .192 & 356.0 & 2006.000 & .208 & 333.2 \\
2002.000 & .199 & 351.0 & 2007.000 & .207 & 328.8 \\
2003.000 & .203 & 346.4 & 2008.000 & .206 & 324.4 \\
\hline
\end{tabular}



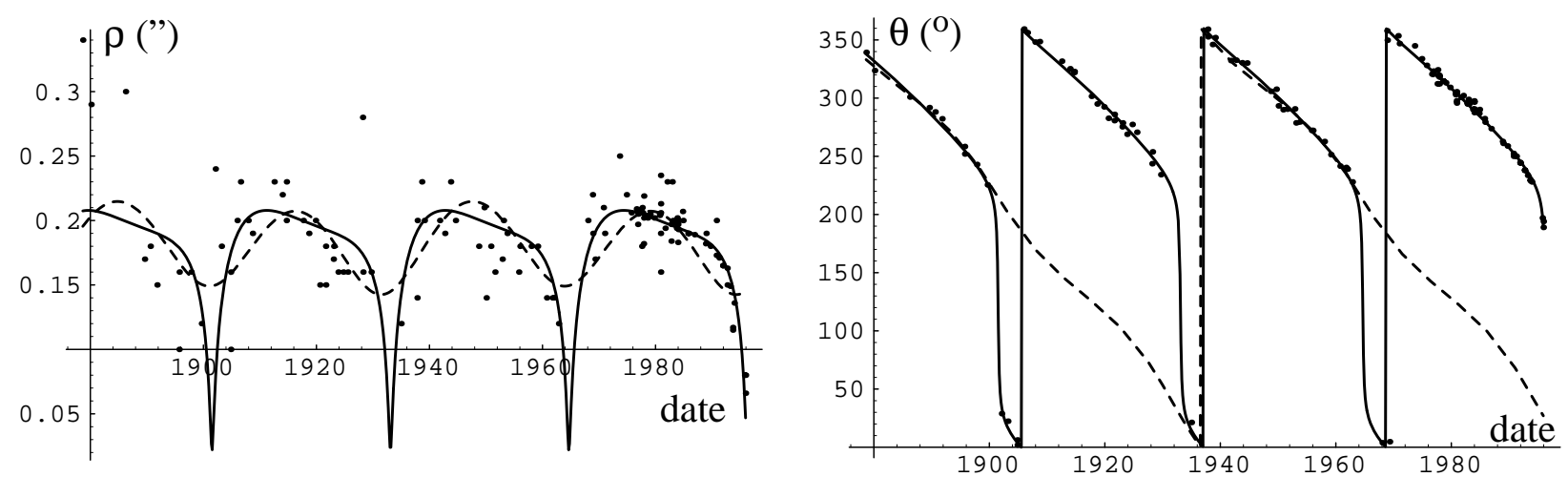

Fig. 2. Separation and angular positions versus date of the couple BU 524 AB. Measurements are represented by the points. The last mesurements are interferometric ones. The solid line is the predicted position from our short-period orbit, dashed lin e is the solution given by our long-period one (see text)

- WDS 11551+4629 - A 1777 - ADS 8347 AB: Our orbit does well fit recent interferometric observations near periastron which present very small residuals. This is another example of the valuable contribution of speckle techniques. The star A is a spectroscopic binary of separation 0.222 mas ( Tokovinin 1997). The star B seems (up to now) single. There are 2 more stars in the quintuple system whose separations are (Tokovinin 1997): $\mathrm{AB} \times \mathrm{C} \simeq 4$ ". and $\mathrm{ABC} \times \mathrm{D} \simeq 63$.'.

\begin{tabular}{|c|c|c|c|}
\hline \multicolumn{4}{|l|}{ Orbital elements } \\
\hline $\bar{~} \overline{\Omega\left({ }^{\circ}\right)(2000.0)}$ & 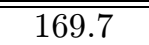 & 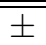 & 4.6 \\
\hline$\omega\left(^{\circ}\right)$ & 26.9 & \pm & 2.1 \\
\hline$i\left(^{\circ}\right)$ & 39.7 & \pm & 1.9 \\
\hline$P(\mathrm{yr})$ & 136.538 & \pm & 8.4 \\
\hline$T(\mathrm{yr})$ & 1987.943 & \pm & 0.41 \\
\hline$e$ & 0.531 & \pm & 0.014 \\
\hline$a$ & $0^{\prime \prime} 225$ & \pm & 0.018 \\
\hline \multicolumn{4}{|c|}{ Astrophysical Information } \\
\hline mag & 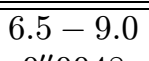 & $\overline{\overline{\mathrm{Sp}}}$ & $\overline{\bar{A} 3 \mathrm{~V}}$ \\
\hline Dynamic parallax & $0 ! 0048$ & & \\
\hline Hipparcos parallax & 0 .'00407 & \pm & $0^{\prime \prime} 00124$ \\
\hline Semi-major axis (AU) & 55.3 & \pm & 21.2 \\
\hline Sum of masses $\left(\mathcal{M}_{\odot}\right)$ & 9.1 & \pm & 11.6 \\
\hline
\end{tabular}

Due to large error bars of semi-major axis $a$ and period $P$, the total mass is very uncertain. For information we found in Tokovinin (1997):

$-\mathcal{M}_{A b}=1.9 \mathcal{M}_{\odot}$

- $\mathcal{M}_{A a}=1.8 \mathcal{M}_{\odot}$

- $\mathcal{M}_{B}=1.5 \mathcal{M}_{\odot}$

giving a total mass $\mathcal{M}_{A}+\mathcal{M}_{B}=5.2 \mathcal{M}_{\odot}$ which agrees with our dynamic parallax.

\begin{tabular}{|ccc|ccc|}
\hline \multicolumn{6}{|c|}{ Ephemeris } \\
\hline \hline Date & $\rho\left(^{\prime \prime}\right)$ & $\theta\left(^{\circ}\right)$ & Date & $\rho\left(^{\prime \prime}\right)$ & $\theta\left(^{\circ}\right)$ \\
\hline 1999.000 & .124 & 287.1 & 2004.000 & .164 & 308.6 \\
2000.000 & .132 & 292.4 & 2005.000 & .171 & 311.7 \\
2001.000 & .140 & 297.2 & 2006.000 & .179 & 314.6 \\
2002.000 & .148 & 301.42 & 2007.000 & .187 & 317.1 \\
2003.000 & .156 & 305.2 & 2008.000 & .194 & 319.5 \\
\hline
\end{tabular}

- Other orbits Orbital elements for the stars A 2681, A 2477, BU 1077, COU 612, COU 321 and STF 2556 are given in Table 3 . These orbits are premature and may be considered as equally tentative as previous ones. Some are based upon a few number of data (17 measurements for A 2681, 20 for Cou 321), some have very small magnitude difference causing quadrant errors. These orbits have to be improved by further observations.

\section{Conclusion}

These observations have shown that the association of the speckle camera of Observatoire Midi-Pyrénées, the ICCD detector of Université de Nice - Sophia Antipolis, and the reduction procedure we have been developing in the last few years is well adapted to binaries study. Despite poor atmospheric conditions, we have obtained a promising list of measurements and started to reach a productive stage in the field of binary stars. In the future we plan to make systematic observations of close double stars resolved by the TBL with unknown, old or uncertain orbits (grade 3 or more in the catalogue Heintz-Worley, Worley \& Heintz 1983). We plan also to observe binaries with close periastron for which observations near the periastron are missing or uncertain. Complementary observations with the speckle camera will also be done with $B V R$ imaging to measure the color indices (we could even obtain the spectra in some favorable cases) of the individual stars for which masses have been determined, in order to derive their spectral class.

Acknowledgements. The authors whish to thank the technical staff of the TBL for assistance during the observations; Michel Aurière (Observatoire Midi-Pyrénées) for help in the preparation of the missions and the Institut National des Sciences de l'Univers for financial support. Thanks are also due to A.A. Tokovinin for help in selecting multiple stars and to Washington Naval Observatory for giving the lists of measurements. This work made use of the SIMBAD 


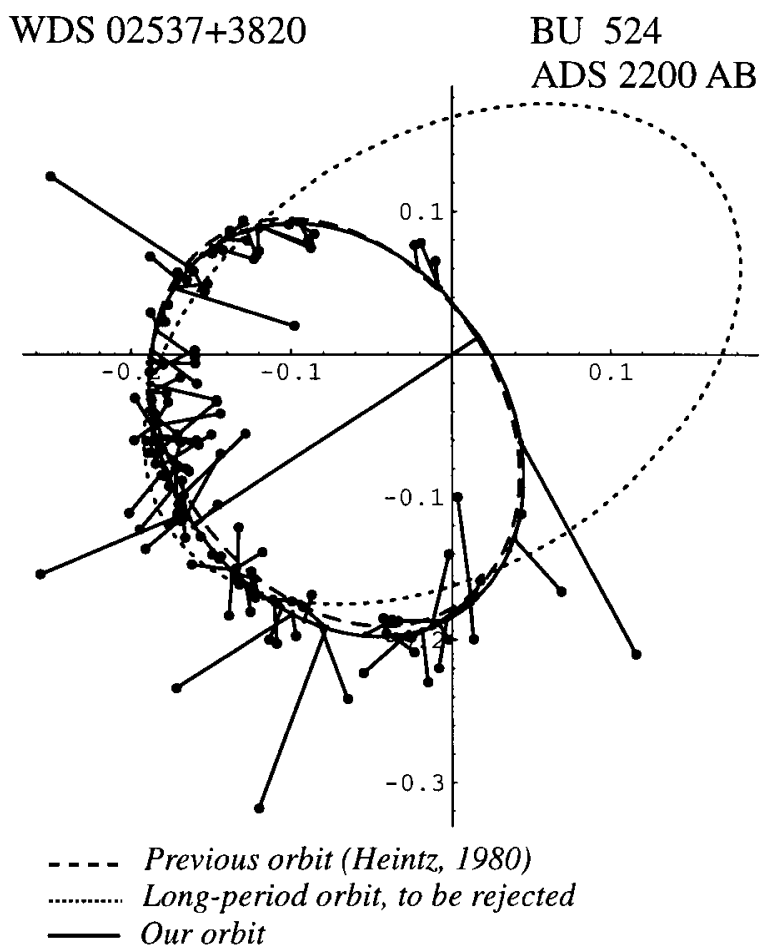

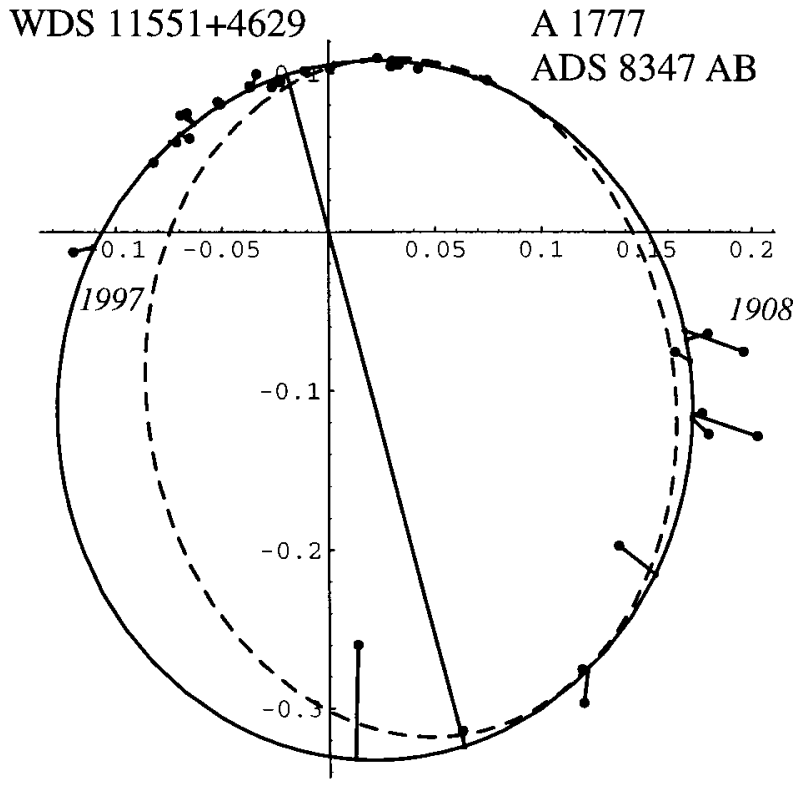

-...-. Previous orbit (Baize, 1991)

Our orbit

Fig. 3. Orbit of BU 524AB and A 1777. Our orbit is plotted in solid line on the graph, previous orbits in dashed line. For BU 524AB the long-period solution is plotted as well (see text). As usual North is down and East is right. Line of apsides of the new orbit is drawn in solid line. Measurements are plotted as points connected to their predicted value ("O-C" lines). Points corresponding the first and last measurement are labelled such as "1971" and "1997"

Table 3. Orbital elements for the 6 stars A 2681 (WDS 06575+0253), A 2477 (WDS 09245+1808), BU 1077 (WDS 11037+6145), Cou 612 (WDS 15390+2545), Cou 321 (WDS 19180+2012) and STF 2556 (WDS 19394+2215)

\begin{tabular}{|c|c|c|c|c|c|c|c|}
\hline WDS & $\Omega\left(^{\circ}\right)$ & $\omega\left(^{\circ}\right)$ & $i\left(^{\circ}\right)$ & $P(\mathrm{yr})$ & $T(\mathrm{yr})$ & $e$ & $a\left(^{\prime \prime}\right)$ \\
\hline $06575+0253$ & 102.6 & 330.1 & 136.8 & 196.96 & 1946.157 & 0.884 & 0.247 \\
$09245+1808$ & 9.8 & 99.1 & 46.6 & 196.67 & 1854.649 & 0.298 & 0.381 \\
$11037+6145$ & $65.3 \pm 16.5$ & $276.2 \pm 19.9$ & $144.1 \pm 22.5$ & $44.637 \pm 0.16$ & $1955.541 \pm 2.1$ & $0.490 \pm 0.13$ & $0.660 \pm 0.040$ \\
$15390+2545$ & 120.4 & 132.9 & 143.7 & 80.306 & 2054.944 & 0.394 & 0.248 \\
$19180+2012$ & 126.0 & 80.0 & 114.0 & 152.3 & 2134.1 & 0.400 & 0.203 \\
$19394+2215$ & 44.5 & 356.0 & 140.9 & 622.4 & 1970.310 & 0.476 & 0.678 \\
\hline
\end{tabular}

astronomical database operated at CDS, Strasbourg, France, and of the CHARA (Georgia State University, Atlanta, Georgia, U.S.A.) 3rd catalogue of interferometric measurements of binary stars.

\section{References}

Aristidi E., Carbillet M., Lyon J.-F., Aime C., 1997a, A\&AS 125,139

Aristidi E., Carbillet M., Prieur J.-L., Lopez B., Bresson Y., Koechlin L., 1997b, A\&AS 126, 555

Baize P., 1981, A\&AS 44, 199

Baize P., 1983, A\&AS 51, 479

Baize P., 1987, A\&AS 71, 177

Baize P., 1988, A\&AS 74, 507

Baize P., 1990, IAU Circ. Inf. 112
Baize P., 1991, A\&AS 87, 49

Baize P., 1991b, IAU Circ. Inf. 115

Baize P., 1991c, IAU Circ. Inf. 114

Baize P., 1992, A\&AS 92, 31

Baize P., 1993, IAU Circ. Inf. 116

Balega I.I., Balega Y.Y., 1988, Sov. Astron. Lett. 14, 393

Barden S.C., 1987, ApJ 317, 333

Carbillet M., Lopez B., Aristidi E., et al., 1996, A\&A 314, 112

Carbillet M., Aime C., Aristidi E., Ricort G., 1998a, A\&AS 127,1

Carbillet M., Lopez B., Aristidi E., et al., 1998b, A\&A 329, 1172

Couteau P., 1978, "L'Observation des étoiles doubles visuelles". Flammarion, Paris

Couteau P., 1990, IAU Circ. Inf. 111

Docobo J.A., Costa J.M., 1985, IAU Circ. Inf. 96

Docobo J.A., Costa J.M., 1988, IAU Circ. Inf. 106 
Hartkopf W.I., Franz O.G., McAllister H.A., 1989, AJ 98, 1014 Hartkopf W.I., Mason B.D., McAllister H.A., 1996, AJ 111, 370

Hartkopf W.I., 1998, Third catalog of interferometric measurements of binary stars (CHARA: Atlanta), http://www.chara.gsu.edu/DoubleStars/Speckle/intro. html

The Hipparcos Catalogue, ESA SP-1200, 1997

Heintz W.D., 1984, A\&AS 56, 5

Heintz W.D., 1988, PASP 100, 834

Heintz W.D., 1996, AJ 111, 408

Hellerich J., 1925, Astron. Nachr. 223, 335

Kowalsky M., 1873, Procès verbaux de l'Université Impériale de Kasan

Mason B.D., Washington Double Star Catalog. USNO: Washington DC

Papaliolios C., Nisenson P., Ebstein S., 1985, Appl. Opt. 24, 287
Popovic G.M., Pavlovic R., 1995a, IAU Circ. Inf. 125

Popovic G.M., Pavlovic R., 1995b, IAU Circ. Inf. 126

Prieur J.-L., Koechlin L., André C., Gallou G., Lucuix C., 1998 (preprint)

Papaliolios C., Mertz L., 1982, Proc. SPIE 331, 360

Scardia M., 1983, Astron. Nachr. 304, 29

Scardia M., 1984a, A\&AS 57, 257

Scardia M., 1984b, Astron. Nachr. 305, 127

Starikova G.A., 1982, Sov. Astron. Lett. 8, 166

Tokovinin A.A., 1996 (private communication)

Tokovinin A.A., 1997, A\&AS 124, 75

Van den Bos W.H., 1938, Union. Obs. Cir. 99, 445

Worden S.P., Murray K.S., Schmidt G.D., Angel J.R.P., 1977, Icarus 32,450

Worley C.E., Heintz W.D., 1983, Pub. U.S. Naval Obs. (2) 24, part VII (available at SIMBAD: http://simbad.ustrasbg.fr/cgi-bin/cdsbib/)

Zulevic D.J., 1990, IAU Circ. Inf. 112 